\title{
Outcomes of Patients with Initially Locally Advanced Pancreatic Adenocarcinoma who did not Benefit from Resection: A Prospective Cohort Study
}

Jonathan Garnier ( $\nabla$ jonathan-garnier@hotmail.fr )

Institut Paoli-Calmettes https://orcid.org/0000-0001-5481-2926

Jacques Ewald

Institut Paoli-Calmettes

Ugo Marchese

Institut Paoli-Calmettes

Marine Gilabert

Institut Paoli-Calmettes

Simon Launay

Institut Paoli-Calmettes

Laurence Moureau-Zabotto

Institut Paoli-Calmettes

Flora Poizat

Institut Paoli-Calmettes

Marc Giovannini

Institut Paoli-Calmettes

Jean-Robert Delpero

Institut Paoli-Calmettes

Olivier Turrini

Institut Paoli-Calmettes

Research article

Keywords: Pancreatic cancer, locally advanced, chemotherapy, chemoradiation, survival

Posted Date: October 4th, 2019

DOI: https://doi.org/10.21203/rs.2.15620/v1

License: (c) (1) This work is licensed under a Creative Commons Attribution 4.0 International License.

Read Full License 
Version of Record: A version of this preprint was published at BMC Cancer on March 12th, 2020. See the published version at https://doi.org/10.1186/s12885-020-6690-1. 


\section{Abstract}

Background: There is no consensus about the optimal treatment for patients with unresectable nonmetastatic locally advanced pancreatic adenocarcinoma (LAPA) after induction chemotherapy. Accordingly, the current study aimed to evaluate the outcomes of patients with LAPA who did not benefit from resection considering the treatment strategy. Methods: Between 2010 and 2017, 234 patients underwent induction chemotherapy for non-metastatic LAPA that could not be treated with surgery. After oncologic restaging, continuous chemotherapy or chemoradiation (CRT) was decided for patients without metastatic disease. The Kaplan-Meier method was used to determine overall survival (OS), and the Wilcoxon test to compare survival curves. Multivariate analysis was performed using the stepwise logistic regression method. Results: FOLFIRINOX was the most common induction regimen (168 patients, $72 \%$ ), with a median of 6 chemotherapy cycles and resulted in higher OS, compared to gemcitabine (19 vs. 16 months, $P=.03$ ); however, no difference was observed after adjusting for age ( $\leq 75$ years) and performance status score (0-1). At restaging, 187 patients $(80 \%)$ had non-metastatic disease: CRT was administered to 126 patients (67\%) while chemotherapy was continued in $61(33 \%)$. Patients who received CRT had comparable characteristics to those who continued with chemotherapy, with similar OS; they also had longer recurrence-free survival (median 13.3 vs. 9.6 months, $\mathrm{P}<.01$ ) and contrastingly, more frequently metastatic disease (hazard ratio $=2.9, \mathrm{P}<.01$ ). Conclusions: The median survival of patients who could not undergo surgery was 19 months. Hence, CRT should not be eliminated as a treatment option and may be useful as a part of optimized sequential chemotherapy to treat both local and metastatic disease.

\section{Background}

Patients diagnosed with non-metastatic locally advanced pancreatic adenocarcinoma (LAPA; comprising both borderline and unresectable tumours) at the time of initial staging receive induction chemotherapy. During the last decade, pancreatic surgeons have been able to perform complex resection/reconstructions in patients with LAPA owing to the benefits of the FOLFIRINOX regimen, which seems to increase the number of patients who finally underwent resection(1). We recently showed that patients with LAPA strongly benefit from resection after induction chemotherapy' even if complex venous resection/reconstruction is needed(2,3). However, most patients who received induction chemotherapy will not have the opportunity to undergo resection because of progressive disease at restaging, altered performance status, or contraindications during explorative laparotomy (i.e. affirmation of extra pancreatic disease or major vasculature invasion). In these patients, chemotherapy and chemoradiation (CRT) are the two commonly used treatments administered by oncologists, in combination with new drugs in the setting of prospective trials. To date, no consensual strategies have been defined in patients who cannot benefit from surgery. Patients with extra-pancreatic disease dissemination at restaging or after laparotomy continue with chemotherapy. However, there is no strong evidence about which treatment is optimal for patients with non-metastatic LAPA. Consequently, multidisciplinary physicians 
have to choose between continuing with chemotherapy (if well tolerated) or CRT according to induction chemotherapy tolerance, patient status, and centre/oncologists preferences.

This study aimed to evaluate the outcomes, according to the treatment, in patients with LAPA who did not benefit from resection after induction chemotherapy.

\section{Methods}

\section{Patient selection}

Between January 1, 2010 and December 31, 2017, 342 patients were diagnosed with LAPA (according to the National Comprehensive Cancer Network guidelines(4)), and received induction chemotherapy at the Institut Paoli-Calmettes, Marseille, France. Among these patients, 234 could not benefit from resection and were included in the study; 108 patients underwent pancreatectomy and their outcomes will be separately reported. Patient data were prospectively entered into a clinical database, as approved by the Institutional Review Board and analysed retrospectively. The study participants provided informed consent, and the study protocol adhered to the tenets of the Declaration of Helsinki.

\section{Initial staging and induction chemotherapy}

All patients had histologically proven pancreatic adenocarcinoma before any treatment. Initial staging consisted of a physical examination, thoraco-abdominal computed tomography (CT), and CA 19-9 serum level determination. Until 2015, patients did not undergo routine liver magnetic resonance imaging (MRI); positron emission tomography (PET) was not performed routinely owing to the lack of evidence of its relevance in this setting. The chemotherapy regimen (i.e. FOLFIRINOX or gemcitabine) was selected according to the patients' age, performance status (PS) score, and decision of the multidisciplinary staff. The FOLFIRINOX regimen comprised a combination of oxaliplatin $\left(85 \mathrm{mg} / \mathrm{m}^{2}\right)$, irinotecan $\left(180 \mathrm{mg} / \mathrm{m}^{2}\right)$, leucovorin, and 5 -fluorouracil (bolus and continuous infusion); the cycle was repeated every 2 weeks. If required, the administration of a 5 -fluorouracil bolus was stopped and oxaliplatin/irinotecan doses were reduced. The gemcitabine regimen comprised a dose of $1000 \mathrm{mg} / \mathrm{m}^{2}$ that was delivered weekly for 3 weeks over subsequent 4-week courses. One cycle corresponded to a 4-week period of treatment. Primary or secondary prophylaxis of neutropenia was initiated using the granulocyte colony-stimulating factor at the physician's discretion.

\section{Treatment after restaging}

All patients were restaged after 3 months of induction chemotherapy to identify patients with severe adverse events and/or disease progression, which helped determine whether patients should continue with the same regimen. After restaging, three strategies were debated among explorative laparotomy, 
continuous chemotherapy with or without drug switching, or CRT. CRT comprised intensity-modulated fractionated radiotherapy combined with concurrent chemotherapy, with capecitabine $\left(800 \mathrm{mg} / \mathrm{m}^{2}\right.$ twice daily, 5 days/week); the total dose of radiotherapy was $54 \mathrm{~Gy}$ in 30 fractions/ 6 weeks. The decision between CRT and continuous chemotherapy with or without drug switching in patients without evidence of extra-pancreatic disease was associated with the time at which the patients were included. Between 2010 and 2015, our strategy was to perform CRT after induction treatment for local treatment in patients without extrapancreatic extension and as part of reserve chemotherapy in case of metastatic progression; however, since 2016, according to the results of the LAP07 randomized trial(5), continuing with chemotherapy was the preferred strategy, with at least 8 cycles of chemotherapy being delivered along with a regimen change if the induction treatment was not well tolerated. The surgical decision for explorative laparotomy was made by the multidisciplinary staff. Resection was not performed in the following cases: a) metastatic spread discovered during explorative laparotomy (i.e., liver metastasis, carcinomatosis, and para-aortic lymph node invasion proven on frozen section(6,7)); b) superior mesenteric artery resection was needed; and c) venous reconstruction was not technically feasible.

\section{Study parameters}

The following variables were evaluated: age, sex, body mass index (BMI), CA 19-9 serum level (at diagnosis and after jaundice resolution), PS score $(0,1,2$, or 3$)$, weight loss $>5 \%$, recent diabetes diagnosis ( $<1$ year), tumour location (i.e. head, body, and tail), back pain, jaundice, arterial invasion at diagnosis, borderline or locally advanced tumours, induction regimen, and continuing with treatment after restaging. The Response Evaluation Criteria in Solid Tumours were not used in our series.

\section{Statistical analysis}

Data analyses were performed using GraphPad Prism software, version 6 (GraphPad Software Inc., La Jolla, CA, USA) and SPSS ${ }^{\circledR}$ version 24 (SPSS Inc., Chicago, IL, USA). Categorical factors were compared using the Fisher's exact test or chi-squared test, and continuous variables using the Student's t-test. The associations between the categorical factors and overall survival (OS) were assessed using the KaplanMeier method (based on the date of diagnosis and censoring date, January 1, 2019); in patients with rapid disease progression and early risk of death, we compared the survival curves using the Wilcoxon test. Multivariate analysis was performed using stepwise logistic regression. Statistical significance was set at $P<.05$.

\section{Result}

Induction treatment, restaging, and strategy after restaging 
The patient characteristics are summarized in Table 1. FOLFIRINOX was the most commonly used induction regimen (168 patients, 72\%), with a median number of 6 cycles of chemotherapy. Patients who received FOLFIRINOX were younger $(P<.01)$ and had a better PS score $(P<.01)$ compared to patients who received gemcitabine (Table 2$)$. At restaging, 187 patients $(80 \%)$ had non-metastatic LAPA whereas 47 (20\%) showed metastatic disease progression. In patients with non-metastatic LAPA, 126 patients (67\%) received CRT whereas 61 (33\%) continued with chemotherapy. Metastatic disease progression was significantly more frequent among patients who received CRT (90 patients, 71\%) than in those who continued with chemotherapy (26 patients, 43\%; $P<.01$ ), with a comparable mean delay (12 vs. 10 months; $P=\mathrm{ns})$.

\section{Survival}

No patient was lost to follow-up (mean follow-up, 31 months). During the study period, 70\% of patients $(n=163)$ had metastatic progression, with the liver being the more frequent site of metastasis (88 patients, $54 \%$ ) followed by carcinomatosis (45 patients, $28 \%$ ). The median survival time of all patients was 18.7 months; the 1-, 3-, and 5-year OS were $80 \%, 7 \%$, and 1\%, respectively. At diagnosis, degraded PS, weight loss, and CA 19-9 level $>500 \mathrm{U} / \mathrm{mL}$ were independent factors that poorly influenced OS (Table 3). At least 8 cycles of chemotherapy provide the optimal OS (19,6 vs. 18,4 months) without reaching the significativity $(p=0,36)$. On comparing induction chemotherapy regimens, FOLFIRINOX resulted in a higher OS than gemcitabine did (19 vs. 16 months, $P=.03$; Figure 1a); however, no difference was observed after adjusting for age ( $\leq 75$ years) and PS score (0-1) (Figure $1 \mathrm{~b})$. At restaging, patients with non-metastatic disease $(n=187)$ who received CRT or continued with chemotherapy had similar patient and tumour characteristics as well as CA 19-9 levels. Although patients who received CRT showed longer recurrencefree survival (median 13.3 vs. 9.6 months, $P<.01$; Figure 2a), they had metastatic disease more frequently (hazard ratio $=2.9,95 \%$ confidence interval [1.5-5.4] $P<.01$ ) and a trend of prolonged OS (median 20 vs. 18 months, $p=0.07$; Figure $2 b$ ) compared to those who continued with chemotherapy.

\section{Discussions}

The current study showed that the clinical status at diagnosis strongly influenced OS rather than the chemotherapy regimen itself, and that at restaging, patients without metastatic disease benefit from receiving CRT.

\section{Patient characteristics and clinical status}

Pancreatic adenocarcinoma often influences the patient's clinical status with weight loss and poor PS score. Our previous study recently showed the importance of these factors in patients with LAPA(8), and 
our results indicated that nutrition and supportive care might help patients receive an optimal therapeutic strategy (i.e. induction treatment and resection). However, this finding needs to be further evaluated, as the patients' recovery course was not as desired considering that a poor clinical status might indicate aggressive tumour biology that cannot be treated with medical support.

At diagnosis, the CA 19-9 serum level and clinical status (PS score and weight loss) were independent factors of poor OS. A high initial CA 19-9 may indicate metastatic disease, with a reduced OS of 12 months(9). Instead of using the CA 19-9 level at diagnosis, the changes in the CA 19-9 level owing to induction chemotherapy might be a useful tool to decide between continuing with chemotherapy (indicated by an increased or still high CA 19-9 serum level) or CRT (indicated by a decrease in the CA 199 serum level)(10). Similarly, the neutrophil-to-lymphocyte ratio(11) and liquid biopsies(12-14), by evaluating circulating tumour cells or circulating tumour DNA, might be helpful to decide between local or systemic treatment at restaging in patients with no evidence of metastatic disease.

\section{Induction treatment}

CRT as induction treatment for patients with LAPA was changed to chemotherapy, because CRT could not be consistently used as a local treatment in a large proportion of patients with unknown metastatic disease $(15,16)$. Thus, gemcitabine was the preferred induction treatment for patients with LAPA until 2010(17), which was the landmark treatment in the FOLFIRINOX era(18). Therefore, the current study focused on patients included in this later period, as we wanted to obtain a clear picture of the patient outcomes in the modern era. As FOLFIRINOX resulted in more patients being able to undergo resection(1), it also provided a survival advantage over the former gemcitabine regimen in our study. However, patients who received gemcitabine induction chemotherapy were older or had a weaker clinical status compared to patients who received the FOLFIRINOX regimen. Moreover, after adjusting for these factors, there was no survival advantage in patients who received the FOLFIRINOX regimen, possibly indicating that the clinical status was more important than the treatment regimen.

\section{Continuing with chemotherapy or CRT at restaging}

Data on the therapeutic strategies and their impact on survival are scarce in patients with unresectable LAPA. In the current study, patients who received CRT at restaging more often developed metastasis, reinforcing the concept that pancreatic cancer is a micrometastatic disease $(5,16)$. Our findings are consistent with those of the LAP07 randomized trial that failed to demonstrate any benefit of CRT in patients with LAPA(5). Considering these points and the related adverse events, it remains unclear if CRT should not be performed for patients with LAPA who did not undergo resection.

Nevertheless, we should not be hasty to conclude that CRT is not beneficial, owing to many reasons. First,

all cases of pancreatic adenocarcinoma show the expression of different genes(19). Thus, some patients 
had a particular tumour biology that confers radiosensitivity. Accordingly, we adopted early neoadjuvant CRT for patients with resectable pancreatic cancer, but we abandoned this strategy owing to disappointing results on considering all treated patients.(20) However, we observed that some resected specimens had a complete histologic response, indicating radiosensitivity. $(21,22)$ Second, in the current study, patients who received CRT had a prolonged recurrence-free survival time and a trend of prolonged OS. This indicates that CRT provided true local control(23), consistent with the loco-regional recurrence rate of $30 \%$ reported previously $(10,19)$. This does not result in a significant OS improvement, as the micrometastatic part of the disease was probably insufficiently treated. Indeed, patients often receive only 4 to 6 cycles of induction chemotherapy before CRT, which therefore needs to be optimized. Third, continuing with chemotherapy results in a rapid increase in the incidence of adverse events, requiring dose reduction or chemotherapy interruption. Thus, CRT might be a therapeutic sequence that provides a systemic break in an occasionally prolonged therapeutic strategy.

\section{Perspectives in patients with non-metastatic LAPA at restaging}

In contrast to the treatment methods available for breast or colorectal cancers, oncologists who treat pancreatic cancer do not have many treatment options at disposal; accordingly, it is not sensible to exclude CRT as an option for patients with unresectable but non-metastatic LAPA at restaging. Therefore, we must focus on restaging to propose the appropriate treatment for each patient, which should include $C T$, liver MRI(24), explorative laparoscopy (with para-aortic lymph node picking if technically feasible), and CA 19-9 serum level measurements. After restaging, patients with no evidence of metastatic disease should have a discussion with multidisciplinary staff considering the following four aspects. First, physicians should consider the tumour evolution after induction treatment, as it makes sense to continue with chemotherapy in patients with an objective response. In this setting, ${ }^{18} \mathrm{~F}$-flurorodeoxyglucose PET(25-27) imaging seemed to be helpful, but the utility has to be validated in large prospective cohorts. Second, the tolerance to induction chemotherapy should be kept in mind, as severe adverse events would require chemotherapy switching or interruption. Third, the number of cycles achieved: our results showed that patients might benefit from at least 8 cycles of chemotherapy before CRT, similar to the results of other studies(10). Finally, the level of CA 19-9 should be considered for the switch to CRT, as the current study showed that a CA 19-9 level $>500 \mathrm{U} / \mathrm{L}$ was an independent predictive factor of survival, indicating persistent tumour activity. Thus, alternating between chemotherapy and CRT might help in treating both local and metastatic disease.

\section{Study limitations}

The present study has some limitations. Owing to the retrospective nature of the study, radiologic assessment of the objective response to induction treatment was not precisely noted. In addition, at the time of restaging, non-metastatic disease was affirmed by using mainly CT and eventually explorative 
laparotomy. Indeed, any patients diagnosed with non-metastatic disease who received CRT probably had an unknown metastatic disease that negatively influenced the OS. Moreover, we did not take into account the toxicity of chemotherapy and objective measures of the quality of life. Nevertheless, the recent period of patient inclusion, the large sample size, and the homogeneity of the studied population are strengths that compensate for these limitations.

\section{Conclusion}

CRT resulted in better disease-free survival and a trend of improved OS over continued chemotherapy for patients with LAPA without any metastasis at restaging who did not benefit from surgery. Thus, CRT should not be completely discarded as a treatment option, as it might be useful when integrated as part of optimized sequential treatment to treat both local and metastatic disease. A low CA 19-9 level, a minimum of 8 cycles of chemotherapy, and controlled disease are factors that can indicate the switch to CRT.

\section{List Of Abbreviations}

$\mathrm{BMI}$, body mass index

CRT, chemoradiation

$\mathrm{CT}$, computed tomography

LAPA, locally advanced pancreatic adenocarcinoma

MRI, magnetic resonance imaging

OS, overall survival

PET, positron emission tomography

PS, performance status

\section{Declarations}

Ethics approval and consent to participate/for publication : The study participants provided informed consent, and the study protocol adhered to the tenets of the Declaration of Helsinki

Availability of data and materials : Patient data were prospectively entered into a clinical database, as approved by the Institutional Review Board and analysed retrospectively

Competing interests : The authors declare that they have no competing interests 
Funding : This research did not receive any specific grant from funding agencies in the public, commercial, or not-for profit sectors.

Authors' contributions : All authors do the same contribution to the manuscript.

Acknowledgements : We would like to thank Editage (www.editage.com) for English language editing.

\section{References}

1. Hackert T, Sachsenmaier M, Hinz U, Schneider L, Michalski CW, Springfeld C, et al. Locally advanced pancreatic cancer: neoadjuvant therapy with Folfirinox results in resectability in $60 \%$ of the Patients. Ann Surg 2016 Sep;264(3):457-63.

2. Al Faraï A, Garnier J, Ewald J, Marchese U, Gilabert M, Moureau-Zabotto L, et al. International Study Group of Pancreatic Surgery type 3 and 4 venous resections in patients with pancreatic adenocarcinoma:the Paoli-Calmettes Institute experience. Eur J Surg Oncol 2019;

3. Bachellier P, Rosso E, Fuchshuber P, Addeo P, David P, Oussoultzoglou E, et al. Use of a temporary intraoperative mesentericoportal shunt for pancreatic resection for locally advanced pancreatic cancer with portal vein occlusion and portal hypertension. Surgery 2014;155(3):449-56.

4. Tempero MA, Malafa MP, Al-Hawary M, Asbun H, Bain A, Behrman SW, et al. Pancreatic Adenocarcinoma, Version 2.2017, NCCN Clinical Practice Guidelines in Oncology. J Natl Compr Canc Netw 2017;15(8):1028-61.

5. Hammel P, Huguet F, van Laethem J-L, Goldstein D, Glimelius B, Artru P, et al. Effect of chemoradiotherapy vs chemotherapy on survival in patients with locally advanced pancreatic cancer controlled after 4 Months of gemcitabine with or without Erlotinib: The LAP07 randomized clinical trial. JAMA 2016;315(17):1844.

6. Schwarz L, Lupinacci RM, Svrcek M, Lesurtel M, Bubenheim M, Vuarnesson H, et al. Para-aortic lymph node sampling in pancreatic head adenocarcinoma: Para-aortic lymph node sampling in pancreatic head adenocarcinoma. Br J Surg 2014;101(5):530-8.

7. Marchese U, Ewald J, Gilabert M, Delpero J-R, Turrini O. Outcomes of pancreatic adenocarcinoma that was not resected because of isolated para-aortic lymph node involvement. J Visc Surg 2018.

8. Duconseil P, Garnier J, Weets V, Ewald J, Marchese U, Gilabert M, et al. Effect of clinical status on survival in patients with borderline or locally advanced pancreatic adenocarcinoma. World J Surg Oncol 2019;17(1).

9. Isaji S, Mizuno S, Windsor JA, Bassi C, Fernández-del Castillo C, Hackert T, et al. International consensus on definition and criteria of borderline resectable pancreatic ductal adenocarcinoma 2017. Pancreatology 2018;18(1):2-11.

10. Truty MJ, Kendrick ML, Nagorney DM, Smoot RL, Cleary SP, Graham RP, et al. Factors predicting response, perioperative outcomes, and survival following total neoadjuvant therapy for borderline/locally advanced pancreatic cancer: Ann Surg 2019;1. 
11. Lee BM, Chung SY, Chang JS, Lee KJ, Seong J. The Neutrophil-Lymphocyte Ratio and PlateletLymphocyte Ratio Are Prognostic Factors in Patients with Locally Advanced Pancreatic Cancer Treated with Chemoradiotherapy. Gut Liver 2018;12(3):342-52.

12. Husain $\mathrm{H}$, Velculescu VE. Cancer DNA in the circulation: The liquid biopsy. Jama 2017;318(13):1272-74.

13. Gemenetzis G, Groot VP, Yu J, Ding D, Teinor JA, Javed AA, et al. Circulating tumor cells dynamics in pancreatic adenocarcinoma correlate with disease status: results of the prospective CLUSTER study. Ann Surg 2018;268(3):408-20.

14. Corcoran RB, Chabner BA. Application of cell-free DNA analysis to cancer treatment. N Engl J Med 2018;379(18):1754-65.

15. Rhim AD, Mirek ET, Aiello NM, Maitra A, Bailey JM, McAllister F, et al. EMT and dissemination precede pancreatic tumor formation. Cell 2012;148(1-2):349-61.

16. Sohal DP, Walsh RM, Ramanathan RK, Khorana AA. Pancreatic adenocarcinoma: treating a systemic disease with systemic therapy. JNCI J Natl Cancer Inst 2014;106(3).

17. Louvet C, Labianca R, Hammel P, Lledo G, Zampino MG, André T, et al. Gemcitabine in combination with oxaliplatin compared with gemcitabine alone in locally advanced or metastatic pancreatic cancer: Results of a GERCOR and GISCAD Phase III Trial. J Clin Oncol 2005;23(15):3509-16.

18. Conroy T, Desseigne F, Ychou M, Bouché O, Guimbaud R, Bécouarn $Y$, et al. FOLFIRINOX versus gemcitabine for metastatic pancreatic cancer. N Engl J Med. 2011;364(19):1817-25.

19. lacobuzio-Donahue CA, Fu B, Yachida S, Luo M, Abe H, Henderson CM, et al. DPC4 gene status of the primary carcinoma correlates with patterns of failure in patients with pancreatic cancer. $\mathrm{J}$ Clin Oncol 2009;27(11):1806-13.

20. Turrini O, Viret F, Moureau-Zabotto L, Guiramand J, Moutardier V, Lelong B, et al. Neoadjuvant 5 Fluorouracil-Cisplatin chemoradiation effect on survival in patients with resectable pancreatic head adenocarcinoma: a ten-year single institution experience. Oncology 2009;76(6):413-9.

21. Moutardier V, Magnin V, Turrini O, Viret F, Hennekinne-Mucci S, Gonçalves A, et al. Assessment of pathologic response after preoperative chemoradiotherapy and surgery in pancreatic adenocarcinoma. Int J Radiat Oncol 2004;60(2):437-43.

22. Turrini O, Viret F, Moureau-Zabotto L, Guiramand J, Lelong B, Bege T, et al. A Modified Dworak Classification Applied to Pancreatic Adenocarcinoma: a Useful Prognostic Factor. Bull Cancer 2007;897-901.

23. Barbier L, Turrini O, Grégoire E, Viret F, Le Treut Y-P, Delpero J-R. Pancreatic head resectable adenocarcinoma: preoperative chemoradiation improves local control but does not affect survival. HPB 2011;13(1):64-9.

24. Marion-Audibert A-M, Vullierme M-P, Ronot M, Mabrut J-Y, Sauvanet A, Zins M, et al. Routine MRI with DWI sequences to detect liver metastases in patients with potentially resectable pancreatic ductal carcinoma and normal liver CT: a prospective multicenter study. Am J Roentgenol 2018;211(5):W217-25. 
25. Kittaka H, Takahashi H, Ohigashi H, Gotoh K, Yamada T, Tomita Y, et al. Role of 18F-

Fluorodeoxyglucose positron emission tomography/computed tomography in predicting the pathologic response to preoperative chemoradiation therapy in patients with resectable T3 pancreatic cancer. World J Surg 2013;37(1):169-78.

26. Ghaneh P, Wong WL, Titman A, Plumpton C, Vinjamuri S, Johnson C, et al. PET-PANC: Multi-centre prospective diagnostic accuracy and clinical value trial of FDG PET/CT in the diagnosis and management of suspected pancreatic cancer. J Clin Oncol 2016;34.

27. Wilson JM, Mukherjee S, Brunner TB, Partridge M, Hawkins MA. Correlation of 18 FFluorodeoxyglucose positron emission tomography parameters with patterns of disease progression in locally advanced pancreatic cancer after definitive chemoradiotherapy. Clin Oncol 2017;29(6):370-7.

\section{Tables}

Table 1: Characteristics of patients with LAPA treated with induction chemotherapy $(N=234)$ 
Mean age (range)

67 (29-89)

Performance status* (\%)

\begin{tabular}{lcc} 
& $0-1$ & $215(92)$ \\
\hline Mean BMI* (range) & $2-3$ & $19(8)$ \\
\hline Weight loss > 5\%* (\%) & $24(14-42)$ \\
\hline Diabetes (\%) & $160(68)$ \\
\hline Back pain* (\%) & $15(36)$ \\
\hline Jaundice (\%) & $152(65)$ \\
\hline CA 19-9 serum level* (UI) (range) & $136(84) * *$ \\
\hline
\end{tabular}

Tumour location (\%)

Head 161 (69)

Body $57(24)$

Tail $16(7)$

Tumour classification (\%)

Borderline $158(68)$

Locally advanced 76 (32)

Arterial invasion* (\%)

$93(40)$

Induction regimen (\%)

\section{FOLFIRINOX 168 (72)}

Gemcitabine $66(28)$

Mean number of cycles before restaging (range) 6 (4-8)

Explorative laparotomy (\%)

45 (19)

Treatment after restaging/laparotomy (\%) 


\section{FOLFIRINOX 78 (33)}

Gemcitabine $30(12)$

Chemoradiation $126(54)$

LAPA: locally advanced pancreatic adenocarcinoma; BMI: body mass index

* at diagnosis; **calculated for patients with head LAPA

Table 2: Characteristics of patients with LAPA at initial staging and according to the delivered induction regimen $(\mathrm{N}=234)$

\begin{tabular}{|c|c|c|c|}
\hline \multicolumn{4}{|c|}{ FOLFIRINOX (n=168) Gemcitabine $(\mathrm{n}=66) P$-value } \\
\hline Mean age $( \pm \mathrm{SD})$ & $64( \pm 9.55)$ & $75( \pm 8.76)$ & $<0.01$ \\
\hline Performance status score $2-3$ (\%) & $8(5)$ & $11(17)$ & $<0.01$ \\
\hline Mean BMI ( $($ SD) & $23.5( \pm 3.81)$ & $23.5( \pm 4.41)$ & ns \\
\hline Weight loss > 5\%* (\%) & $110(65)$ & $50(76)$ & ns \\
\hline Diabetes (\%) & $10(6)$ & $5(7.5)$ & ns \\
\hline Back pain (\%) & $108(64)$ & $44(67)$ & ns \\
\hline CA 19-9 serum level* (UI) ( $($ SD) & $3884( \pm 13137)$ & $1743( \pm 4916)$ & ns \\
\hline Tumour location (\%) & & & ns \\
\hline Head & $117(70)$ & $44(65)$ & \\
\hline Body & $40(24)$ & $17(26)$ & \\
\hline Tail & $11(6)$ & $5(9)$ & \\
\hline Tumour classification (\%) & & & ns \\
\hline Borderline & $116(69)$ & $42(64)$ & \\
\hline Locally advanced & $52(31)$ & $24(36)$ & \\
\hline Arterial invasion (\%) & $68(40.5)$ & $25(38)$ & ns \\
\hline
\end{tabular}


Table 3: Univariate and multivariate analyses of factors influencing overall survival at diagnosis $(\mathrm{N}=234)$

\begin{tabular}{lccc} 
& $\boldsymbol{P}$-value & Hazard Ratio & $\boldsymbol{P}$-value \\
\hline Performance status score $\geq \mathbf{2}$ & $<0.01$ & $1.92[1.06 ; 3.46]$ & $\mathbf{0 . 0 3 1}$ \\
\hline Borderline status at diagnosis & 0.35 & $\mathrm{~ns}$ \\
\hline Arterial invasion at diagnosis & 0.48 & $\mathrm{~ns}$ \\
\hline Back pain & 0.041 & $\mathrm{~ns}$ \\
\hline Weight loss & 0.037 & $1.49[1.02 ; 2.19]$ & $\mathbf{0 . 0 4 1}$ \\
\hline CA 19-9 >500 & 0.087 & $1.79[1.25 ; 2.57]$ & $<0.01$ \\
\hline Continuous chemotherapy & 0.065 & $\mathrm{~ns}$
\end{tabular}

\section{Figures}


Figure 1.

a) All patients

b) Matched patients aged $\leq 75$ years and PS $0-1$

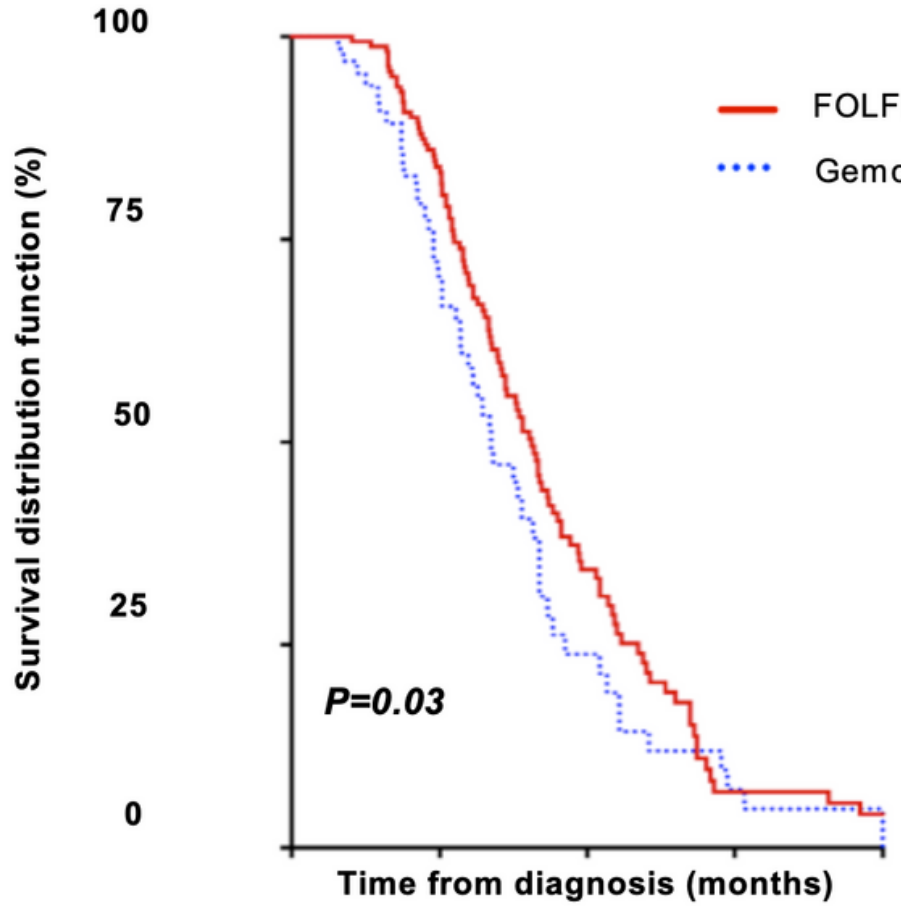

$\begin{array}{llllll}\begin{array}{l}\text { Patients at risk } \\ \text { (months) }\end{array} & 0 & 12 & 24 & 36 & 48 \\ \text { FX } & 168 & 121 & 34 & 6 & 4 \\ \text { Gem } & 66 & 39 & 11 & 4 & 2\end{array}$

NOX (FX)

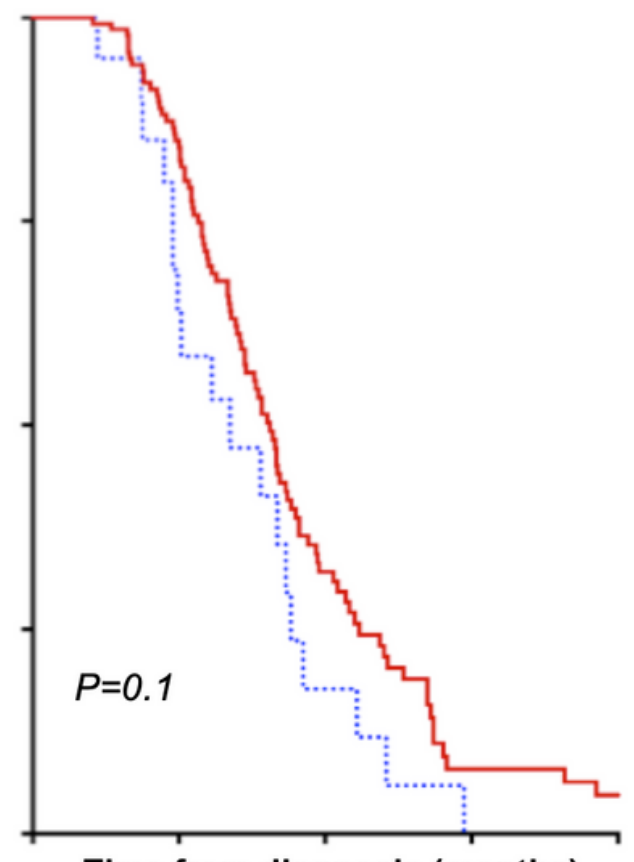

Time from diagnosis (months)

$\begin{array}{lllll}0 & 12 & 24 & 36 & 48\end{array}$

$\begin{array}{lllll}142 & 105 & 29 & 6 & 4\end{array}$

$\begin{array}{lllll}20 & 13 & 4 & 2 & 1\end{array}$

Figure 1

Overall survival of patients with LAPA who received FOLFIRINOX or gemcitabine as induction chemotherapy: a) overall survival of all patients, and $b$ ) overall survival of patients aged $\leq 75$ years and performance status (PS) score of 0-1. LAPA, locally advanced pancreatic adenocarcinoma 
Figure 2.

a) Recurrence-free survival

b) Overall survival

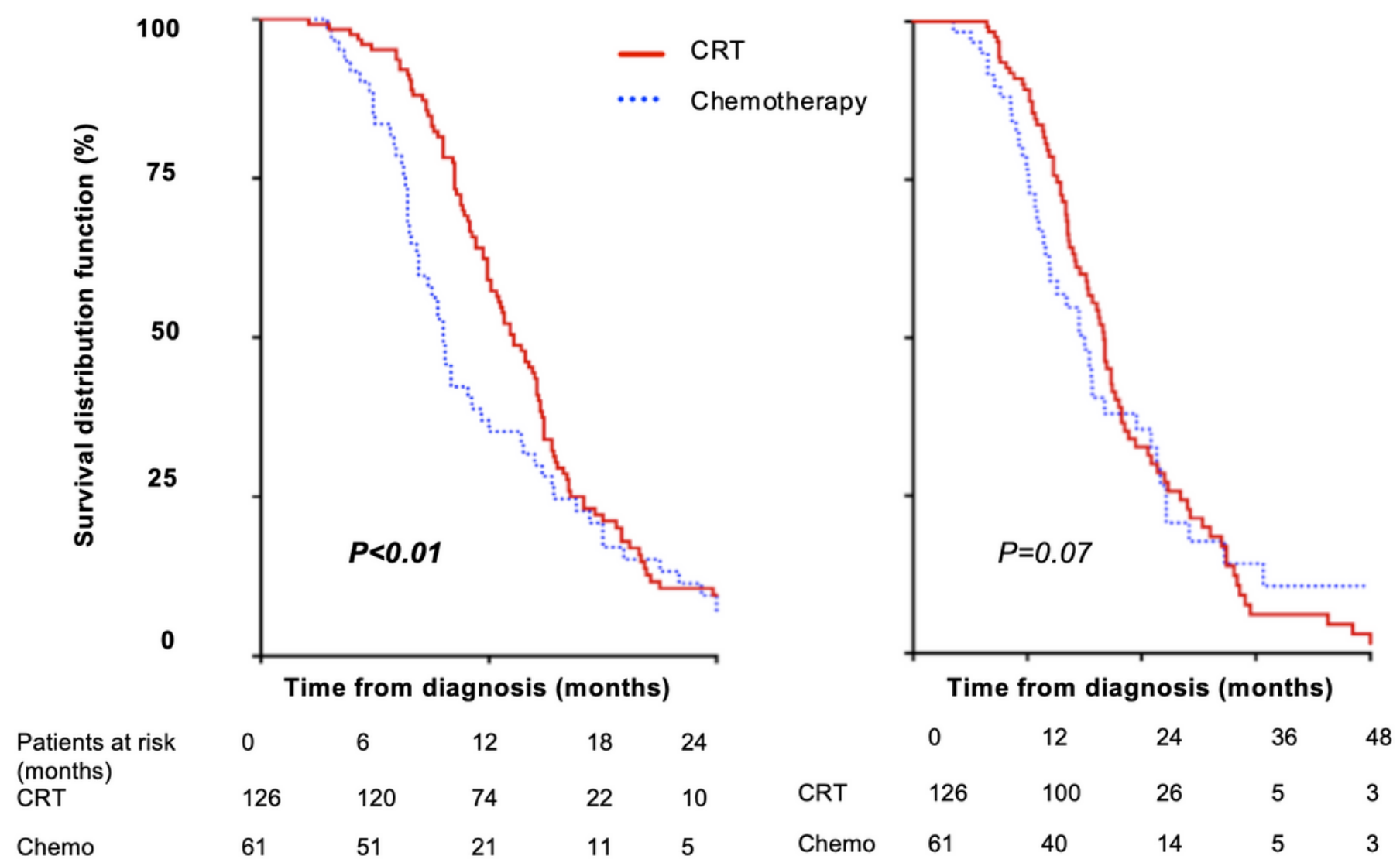

Figure 2

Survival of patients with non-metastatic LAPA at restaging $(n=187)$ who received CRT or continued chemotherapy. LAPA, locally advanced pancreatic adenocarcinoma; CRT, chemoradiation 